\title{
Detection of forest fires outbreaks by dynamic fuzzy logic controller
}

JOSUÉ TOLEDO-CASTRO*, Department of Computer Engineering and Systems, University of La Laguna, 38271 Tenerife, Spain.

NAYRA RODRíGUEZ-PÉREZ**, Department of Computer Engineering and Systems, University of La Laguna, 38271 Tenerife, Spain.

PINO CABALLERO-GIL ${ }^{\dagger}$, Department of Computer Engineering and Systems, University of La Laguna, 38271 Tenerife, Spain.

IVáN SANTOS-GONZÁLEZ ${ }^{\dagger \dagger}$, Department of Computer Engineering and Systems, University of La Laguna, 38271 Tenerife, Spain.

CANDELARIA HERNÁNDEZ-GOYA ${ }^{\S}$, Department of Computer Engineering and Systems, University of La Laguna, 38271 Tenerife, Spain.

RICARDO AGUASCA-COLOMO ${ }^{\S}$, Instituto Universitario de Sistemas Inteligentes y Aplicaciones Numéricas en Ingeniería, University of Las Palmas de Gran Canaria, 35017 Gran Canaria, Spain.

\begin{abstract}
The use of wireless sensor networks (WSNs) and the Internet of things to detect forest fire outbreaks may help to reduce the response time and avoid natural disasters. This work proposes the deployment of WSN to enhance the real-time monitoring of dynamic variables such as polluting gases, temperature or the presence of fire flames by infrared. In addition, the activation of forest fire alerts if environmental status may involve evidence of a recent fire outbreak. A fuzzy-based controller is implemented to manage the uncertainty related to estimating forest fire occurrence and dynamic update of fuzzy sets is developed to distinguish usual and unusual environmental conditions of every monitored forest area. Merkle trees, AES and hash functions are considered to protect the integrity of environmental data.
\end{abstract}

Keywords: Fuzzy logic, forest fires, WSN, Internet of things

*E-mail: jtoledoc@ull.edu.es

**E-mail: mrodripe@ull.edu.es

†E-mail: pcaballe@ull.edu.ess

t†E-mail: jsantosg@ull.edu.es

$\S_{\text {E-mail: mchgoya@ull.edu.es }}$

$\S \S$ E-mail: ricardo.aguasca@ulpgc.es

Vol. 00, No. 0, (C) The Author(s) 2020. Published by Oxford University Press. All rights reserved. For permissions, please e-mail: journals.permission@oup.com.

doi:10.1093/jigpal/jzaa036 


\section{Detection of Forest Fires Outbreaks}

\section{Introduction}

Most of the forest fires generated are the result of human activity today. It is necessary to promote technological innovation in the environmental management of forest areas to promote the prevention and detection of forest fires. In Spain, the number of forest fires that occur every year is quite high. Their early detection is essential to prevent the occurrence of large forest fires. According to the Ministry of Agriculture, Fisheries and Food of Spain government, an average annual number of 12573 accidents occurred in the decade between the 2008 and 2017. The preliminary statistical revision of 2018 shows 7143 incidents, of which 1989 were forest fires [1].

There are different variables or dynamic risk factors that can alert at the beginning of a new fire. These include the increase in polluting gases typically released by the burning of plantbased fuels whose hydric stress, caused by climate conditions in forest areas over time, severely affects. Nowadays, the annual global average concentration of $\mathrm{CO}_{2}$ in the atmosphere exceeds $400 \mathrm{ppm}$ [2]. Technological innovation in the field of wireless sensor network (WSN) [3] and the Internet of thing (IoT) [4] has made the monitoring of a large number of variables across different areas and environments possible. Among these variables, the polluting gases or the environmental temperature highlights. The progressive and unusual increase in temperature values in a forest area can also indicate the beginning of the combustion process of plant-based fuels. Likewise, there are other types of sensors that are quite useful for fires detection allowing fire flames to be detected on the basis of infrared technology in the proximity where the sensor is located. The development of an IoT prototype that integrates these sensors would allow detecting the presence of fire flames at a $360^{\circ}$ angle. To this respect, different communication protocols and network architectures can be implemented to ensure the connectivity of the sensor nodes and the integrity, confidentiality and authenticity of the measured environmental information. However, the interpretation of environmental information measured by WSN for the activation of fire alerts can provide uncertainty and inaccuracy. The application of fuzzy logic-based methods can make the process of classifying and evaluating the status of the monitored environmental variables more efficient by expressing a level of membership of the data with different possible fuzzy sets, in contrast to classical logic. Mamdani inference or the Sugeno methods stand out [5].

The system proposed here implements a forest fire controller in charge of detecting forest fire outbreaks on the basis of analysing environmental variables and applying fuzzy logic techniques, such as the Mamdani inference. For this purpose, the existence of high concentrations of polluting gases, increases in temperature values or unusual light intensity jointly with detecting fire flames by infrared are monitored in real time using WSN. Fuzzy sets are dynamically updated depending on the usual environmental conditions of every monitored forest area.

This work is structured as follows. Section 2 describes the related work. The proposed system and the security methods are detailed in Section 3. Then, the fuzzy-based controller implemented is explained in Section 4. Finally, Section 5 includes some conclusions, work in progress and future works.

\section{Related work}

Nowadays, designing algorithms that could help in the detection of forest fires is a challenge. The advancement of new technologies makes possible the application of different methods that allow the detection of forest fires. To this respect, Mahmoud and Ren's work [6] proposed a video-based image processing system to identify the presence of forest fires and avoid false alarms. Regarding unmanned aerial vehicle-based systems, a survey is proposed by Yuan et al. [7]. On the other hand, 
Yu et al. [8] proposed a neural network method to process network data through different proposed simulations with the aim of detecting fire. Molina-Pico et al.'s work [9] also highlighted and described a hierarchical network of wireless sensors for the early detection of fires in areas at risk.

WSN usually have limited computing capacity, so the applied security algorithms must be adapted to this limitation. In addition to this, the integrity of information transmitted between nodes must be ensured. Martins and Guyennet's work [10] detailed a survey related to some attacks and security mechanisms carried out in WSN. Similarly, Gao et al.'s [11] proposed a system to save resources on the IoT devices that generally compose WSN. The use of cryptography techniques ensures the security of services provided by IoT devices in the work of Choo et al.'s [12]. On the other hand, fuzzy logic is applied in systems with the aim of improving the management of uncertainty and inaccuracy related to the information managed by a system. Amri et al. [13] applied this technique for the location of WSN nodes. Furthermore, fuzzy logic can be also applied to analyse security processes and the existence of risks related to accident scenarios [14]. With regard to detect forest fires, several approaches provide fuzzy logic systems based on using environmental variables. Temperature, humidity, smoke, polluting gases or light stands out as their linguistic variables. They may provide evidence of unusual environmental changes $[15,16]$. Other works propose decision systems based on integrating fuzzy models for long-term fire risk estimation [17].

With regard to other works, the system proposed in this work analyses dynamic environmental variables useful in fire detection, such as the detection of unusual polluting gases, temperature increases or nearby fire flames by infrared sensors at a range of $360^{\circ}$. The variation time between unusual measurements is also monitored. A specific WSN architecture is provided to measure environmental variables in real-time and a fuzzy-based controller analyses this information to evaluate the occurrence of forest fire outbreaks. Fuzzy sets are dynamically updated to classify input data as usual or unusual on the basis of the historical results provided by the fuzzy-based controller. Communications between WSN nodes and the Cloud Server are securely performed by encrypting the transmitted data, in addition to ensuring its integrity by applying hashes and authentication methods for WSN nodes.

\section{WSN architecture}

This system proposes the deployment of a WSN through forest areas of interest with the aim of detecting outbreaks of a forest fire. A prototype of IoT device is implemented for the proposed WSN. Several useful environmental sensors are assembled and intended to detect the presence or fire flames, abrupt variations of light intensity and unusual measurements of temperature and polluting gases $\left(\mathrm{CO}_{2}\right.$ and $\left.\mathrm{CO}\right)$. Thus, each WSN node performs real-time monitoring of dynamic environmental variables in the forest area in which it is located at $360^{\circ}$. As Figure 1 shows, the proposed WSN follows a tree-based topology network. The forest area to be monitored is divided into quadrants. Regarding a specific colour code, each of these quadrants shows the status of dynamic environmental variables and the probabilities related to the beginning of a nearby outbreak of fire.

This colour code is composed of green (low fire detection probabilities (LFDP)), yellow (medium fire detection probabilities (MFDP)), orange (high fire detection probabilities (HFDP)) and red (extreme fire detection probabilities (EFDP)). Every environmental package registered by the WSN is analysed by a fuzzy-based forest fire controller detailed in Section 4. This controller estimates the probabilities of forest fire outbreaks with respect to every monitored forest area aiming at enabling forest fire alerts and updating the environmental map integrated into the developed Web and the mobile applications. 


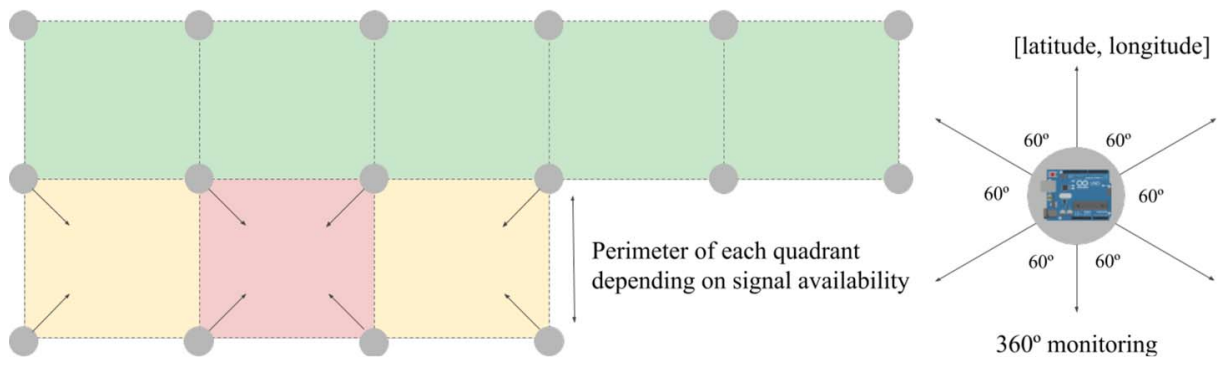

FIGURE 1. WSN distribution scheme.

\subsection{IoT prototype, electronics and sensors}

Every WSN node is based on a specific IoT device implemented and composed of the main board Arduino Mega 2560, several environmental sensors and wireless communication modules (4G and Wi-Fi) aimed to allow communications between WSN nodes and the Cloud Server. The energy selfsufficiency of the device is provided by assembling a solar panel and lithium-based rechargeable batteries.

Regarding the environmental measuring process, every IoT device contains 6 infrared-based fire flame sensors that are assembled following a ring structure, so enabling the detection of forest fire flames at $360^{\circ}$. Light-sensitive photoresistors are also integrated to evaluate unusual changes in light intensity at nights due to nearby fire flames. On the other hand, the analogue sensors MQ-135 and MQ-9 are considered to monitor the air quality of the forest area with the aim of detecting smoke and unusual increase of polluting gases emitted because of burning plant-based fuels. Finally, an infrared-based temperature sensor is integrated to monitor temperature measurements and unusual increases (see Figure 2).

These Arduino-based WSN nodes are coordinated by gateways constituted by Raspberry Pi devices in charge of managing their environmental measurement cycle depending on the previous probabilities of forest fire outbreaks provided by the fuzzy controller. Thus, two-way communications between coordinators and Arduino microcontrollers are enabled.

\subsection{Security scheme for WSN}

Figure 3 shows the security methods applied to ensure the integrity, confidentiality and authentication of environmental measurements registered by WSN. The implementation of some security methods highlights, such as the use of Merkle Trees and SHA-3 signature for signing environmental packages, Transport Layer Security for communications with the Cloud Server and AES Cipher Block Chaining.

\section{Fuzzy-based fire detection method}

The fuzzy logic is used to implement a forest fire detection controller aimed to enhance the management of inaccuracy and uncertainty related to forest fire risk analysis and the detection of new outbreaks of fire (see Figure 4). For this purpose, Mamdani's inference method is applied, so distinguishing 4 main steps: fuzzification, inference engine, aggregation of outputs and defuzzification. To this respect, the implemented fuzzy-based controller constitutes a complementary tool for 


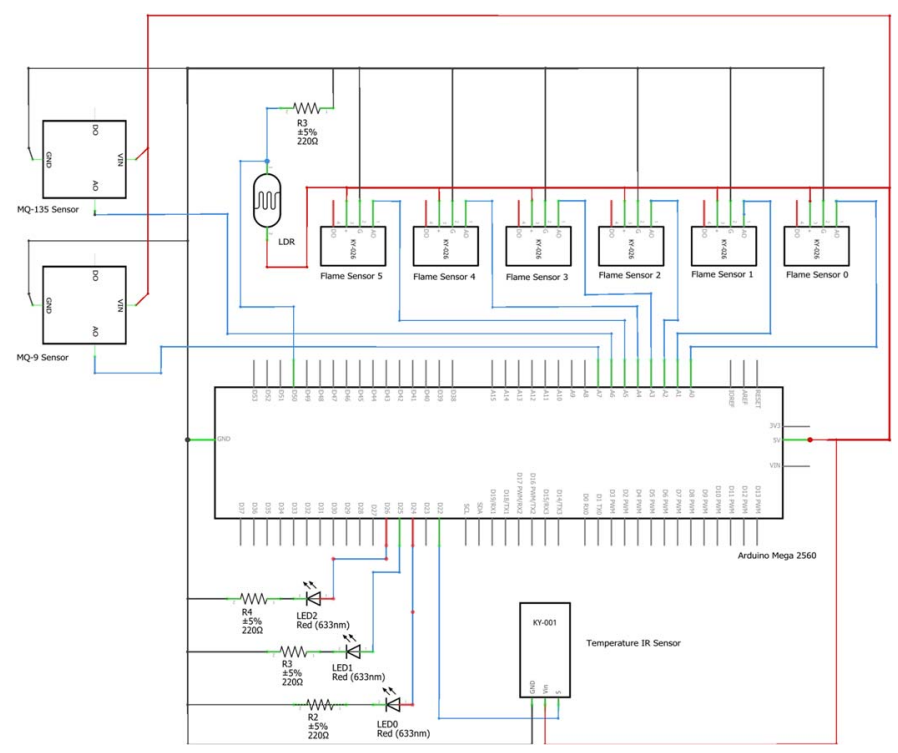

FIGURE 2. IoT device schematics.

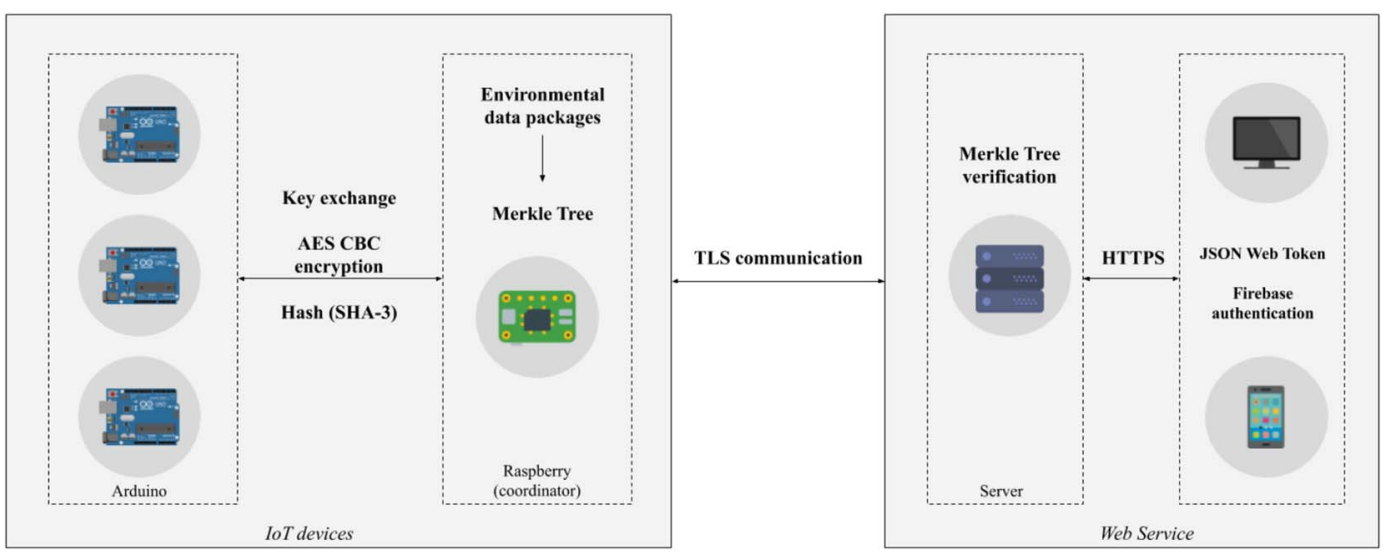

FIGURE 3. Security scheme.

the emergency corps, so that it is aimed to make efficient the monitoring of dynamic environmental factors, activating forest fire alerts and reduce the response time.

Table 1 shows the notation used to explain fuzzy-based controller of fire outbreaks.

\subsection{Linguistic variables}

The following list details the input linguistic variables considered for the proposed fuzzy-based fire controller:

1. Environmental dynamic variables monitored by WSN: temperature (temperature), carbon dioxide (CO2) and carbon monoxide $(\mathrm{CO})$ as polluting gases (increased as a result of the 


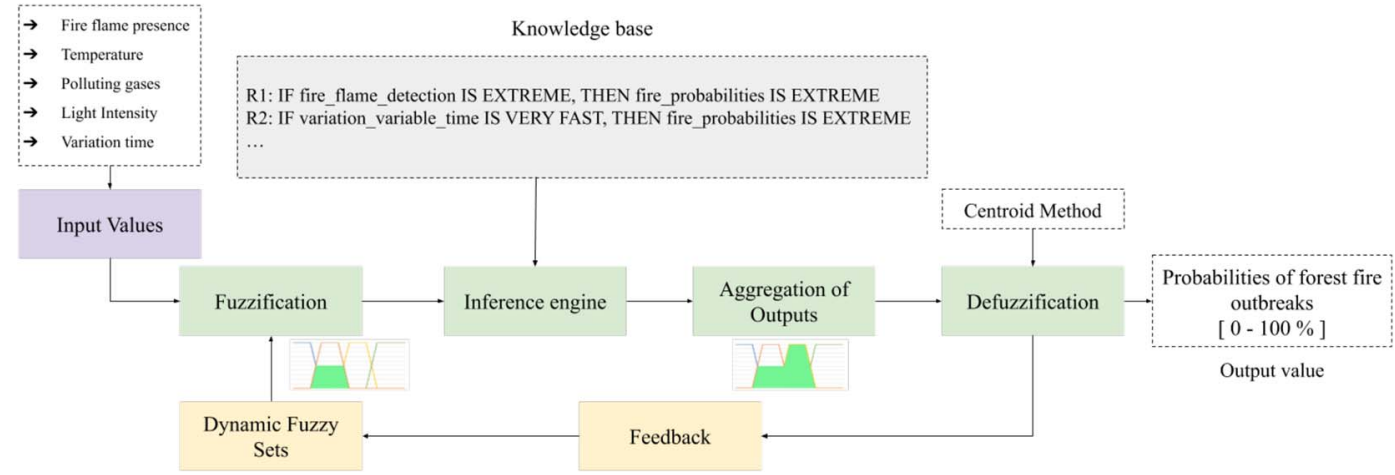

FiguRE 4. Fuzzy-based forest fire controller

TABLE 1. Notation fuzzy-based controller.

\begin{tabular}{|c|c|}
\hline Notation & Meaning \\
\hline$v$ & $\begin{array}{l}\text { Linguistic variable proposed for this fuzzy-based controller. It } \\
\text { can take the following values: temperature, } C O 2, C O \text {, } \\
\text { fire_flame_detection, fire_flame_light, time_of_day, } \\
\text { variable_variation_time. }\end{array}$ \\
\hline$m_{v}$ & $\begin{array}{l}\text { Measurement of linguistic variable } v \text { that constitutes an input valu } \\
\text { of the fuzzy-based forest fire controller }\end{array}$ \\
\hline$\mu_{\text {fuzzy_set }_{v}(i)}\left(m_{v}\right)$ & $\begin{array}{l}\text { The fuzzy value of the input value } m_{v} \text { according to the i-th fuzzy } \\
\text { set proposed for the linguistic variable } v\end{array}$ \\
\hline fuzzy_domain(v) & Fuzzy domain proposed for linguistic variable $v$ \\
\hline$(\max \mid \min v a l u e)$ fuzzy_domain $v$ & Maximum/minimum value of v's fuzzy domain \\
\hline fuzzy $_{\text {set }_{v}}(i)$ & $i$-th fuzzy set proposed for the linguistic variable $v$ \\
\hline $\operatorname{maxHV}\left(\right.$ fuzzy $_{\text {set }_{v}}($ Usual $\left.)\right)$ & $\begin{array}{l}\text { Maximum historical value; maximum discrete value with a } 100 \% \\
\text { level of membership with respect to the fuzzy set usual defined } \\
\text { for variable } v\end{array}$ \\
\hline $\operatorname{minHV}\left(\right.$ fuzzy $_{\text {set }_{v}}($ Usual $\left.)\right)$ & $\begin{array}{l}\text { Minimum historical value; minimum discrete value with a } 100 \% \\
\text { level of membership with respect to the fuzzy set usual defined } \\
\text { for variable } v\end{array}$ \\
\hline variation_interval $_{v}$ & $\begin{array}{l}\text { Variation interval of linguistic variable } v \text {. It is aimed to analyse } \\
\text { increases and decreases of the variable } v \text { from the moment when } \\
\text { unusual measurements are registered by a WSN node. Thus, this } \\
\text { interval is used to define triangular-based fuzzy sets for unusual } \\
\text { measurement classification with the aim of analysing in detail } \\
\text { variations of fuzzy values over time (see Table 2). }\end{array}$ \\
\hline
\end{tabular}

burning process of plant-based fuels). The input values for the inference engine are the last measurement, the second last measurement and the average of every variable.

2. Light intensity (fire_flame_light). Measured by light-sensitive photoresistors assembled to each WSN IoT node. Like the previous case, the last measurement, the second last measurement and the average are used. This variable is considered by the inference engine depending 


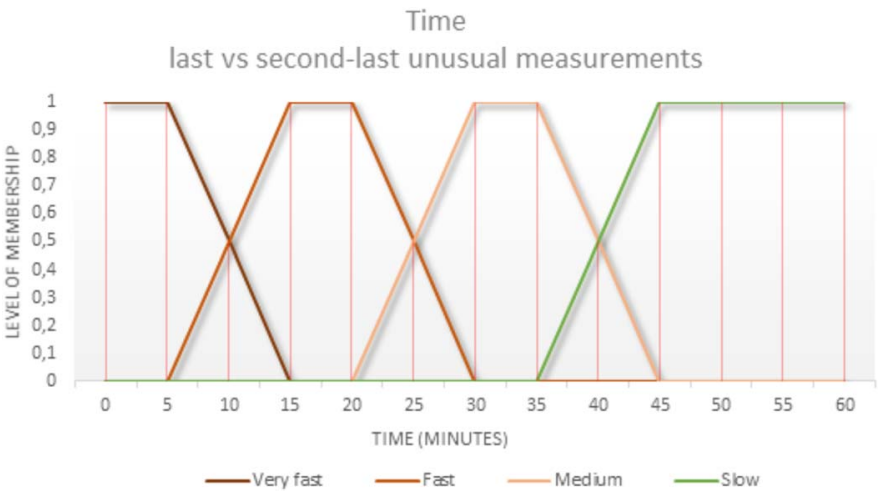

FIGURE 5. Time between different fuzzy values of unusual measurements

on the time of day in which it is analysed. To this respect, the time of day is also treated as a linguistic variable (time_of_day) whose membership functions determine fuzzy values 'daylight' or 'nightlight' with regard to a particular input time data. This variable can be useful in night periods to detect unusual light changes caused by a close fire flame. However, during the daytime, the natural sunlight makes determining with certainty that a change in measured light intensity is caused by a nearby flame difficult.

3. For each linguistic variable, the time elapsed (variable_variation_time) between the last registered measurement and a previously registered measurement whose corresponding fuzzy values are different. This linguistic variable is only evaluated by inference rules if the fuzzy value corresponding to the last environmental measure presents high membership levels with fuzzy sets different from usual (fuzzy set defined for every input linguistic variable for classifying usual measurements). Therefore, indicating that the environmental measurements are not usually registered in the forest area where the WSN node is located. This linguistic variable is used to evaluate the increase in the probabilities related to the occurrence of forest fire outbreaks based on the variations over time of the environmental linguistic variables (see Figure 5).

4. Fire flame detection (fire_flame_detection). This linguistic variable detects by infrared the existence of fire flames on the basis of an angle of $60^{\circ}$ according to the direction where the sensor is located. If the value returned by the flame presence sensor integrated into every WSN node is closer to 0 , then there is a greater probability of a nearby flame. Like the aforementioned linguistic variables, the membership function implemented classifies the measurement of fire flame presence depending on whether it is a usual or unusual measurement with regard to the forest area where the corresponding sensor is located.

Figure 6 shows the proposed generic form for the membership functions of temperature, $\mathrm{CO}_{2}$, $\mathrm{CO}$, light intensity due to fire flames and the presence of fire flames. For these variables, the fuzzy set usual highlights and it is intended to classify all environmental measurements considered usual or normal in the forest area where every WSN node is located. It means that these types of measurements do not have historically provided medium, high or extreme probabilities of forest fire outbreaks. This fuzzy set is dynamically updated with regard to each monitored zone and WSN IoT device according to the defined Feedback process. This is supplied from the outputs obtained by the fuzzy controller with each new environmental information package analysed. These outputs 


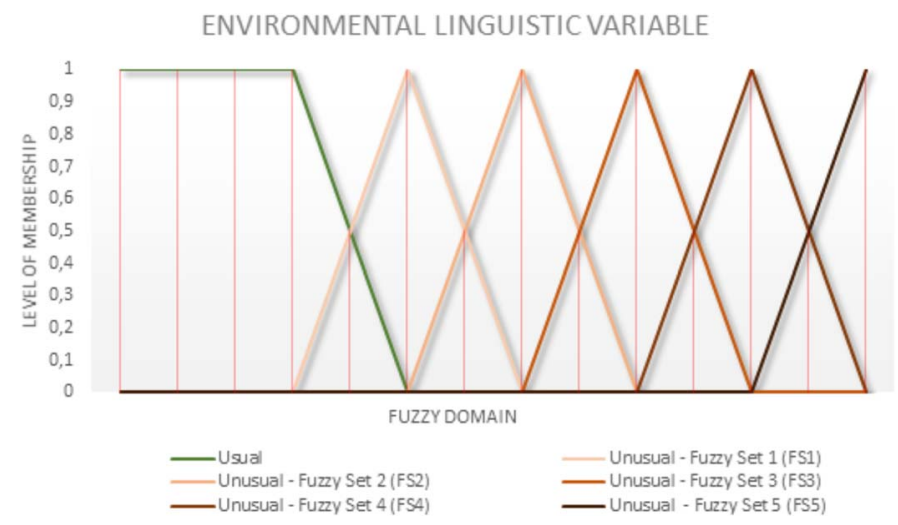

FIGURE 6. Usual fuzzy set and triangular-based unusual fuzzy sets

TABLE 2. Variation interval defined for input linguistic variables.

\begin{tabular}{ll}
\hline Linguistic variable & variation_interval $_{v}$ \\
\hline Temperature & $5^{\circ} \mathrm{C}$ \\
$\mathrm{CO} 2$ & $50 \mathrm{ppm}$ \\
$\mathrm{CO}$ & $10 \mathrm{ppm}$ \\
Light Intensity & 100 (raw sensor data) \\
Fire flame presence & 50 (raw sensor data) \\
\hline
\end{tabular}

define the probabilities that a recent fire has been generated in the proximity of the sensor. Thus, this process updates one maximum ( $\operatorname{maxHV}$ ) and one minimum $(\operatorname{minHV})$ environmental historical value or measurement with a membership level of $100 \%$ with the usual fuzzy set. Because each IoT node is located in a different forest area characterized by different environmental conditions, a different and specific usual fuzzy set is updated for each of them.

For each environmental linguistic variable considered (temperature, $\mathrm{CO}_{2}, \mathrm{CO}$, etc.), $\boldsymbol{n}$ triangularbased fuzzy sets (denoted as unusual-fuzzy set $i$-th from $i=1$ to $i=n$ ) are added to their corresponding membership functions aimed to classify unusual measurements in the monitored forest areas. The amplitude of each one of these triangular fuzzy sets is defined according to the variation interval defined for each linguistic variable (see Table 2). Once the environmental measurements of a linguistic variable are unusual with regard to a monitored forest area, it is possible to classify their changes (increase and decrease) through the corresponding membership functions. To this respect, the proposed inference engine can evaluate the aggravation of the status of an environmental variable with respect to the increased probabilities of a forest fire. The following equation shows the fuzzification process of a measurement $m_{v}$ according to a triangular-based unusual fuzzy set (taking into account $\boldsymbol{n}$ triangular sets calculated).

$$
\begin{gathered}
\forall\left(m_{v}>\operatorname{maxHV}\left(\text { fuzzy_set }_{v}\left(U_{\text {sual })}\right)\right)\right. \\
\exists n \in \mathbb{N}\left|n=\frac{\mid(\max \text { value }) \text { fuzzy_domain }_{v}-\operatorname{maxHV}_{v} \mid}{\text { variation_time }_{v}}, \exists i \in \mathbb{N}\right| 1 \leq i \leq n \\
\Rightarrow \mu_{\text {fuzzy_set }_{v}(\text { Unusual_FS }(i))\left(m_{v}\right)>0 \%}
\end{gathered}
$$



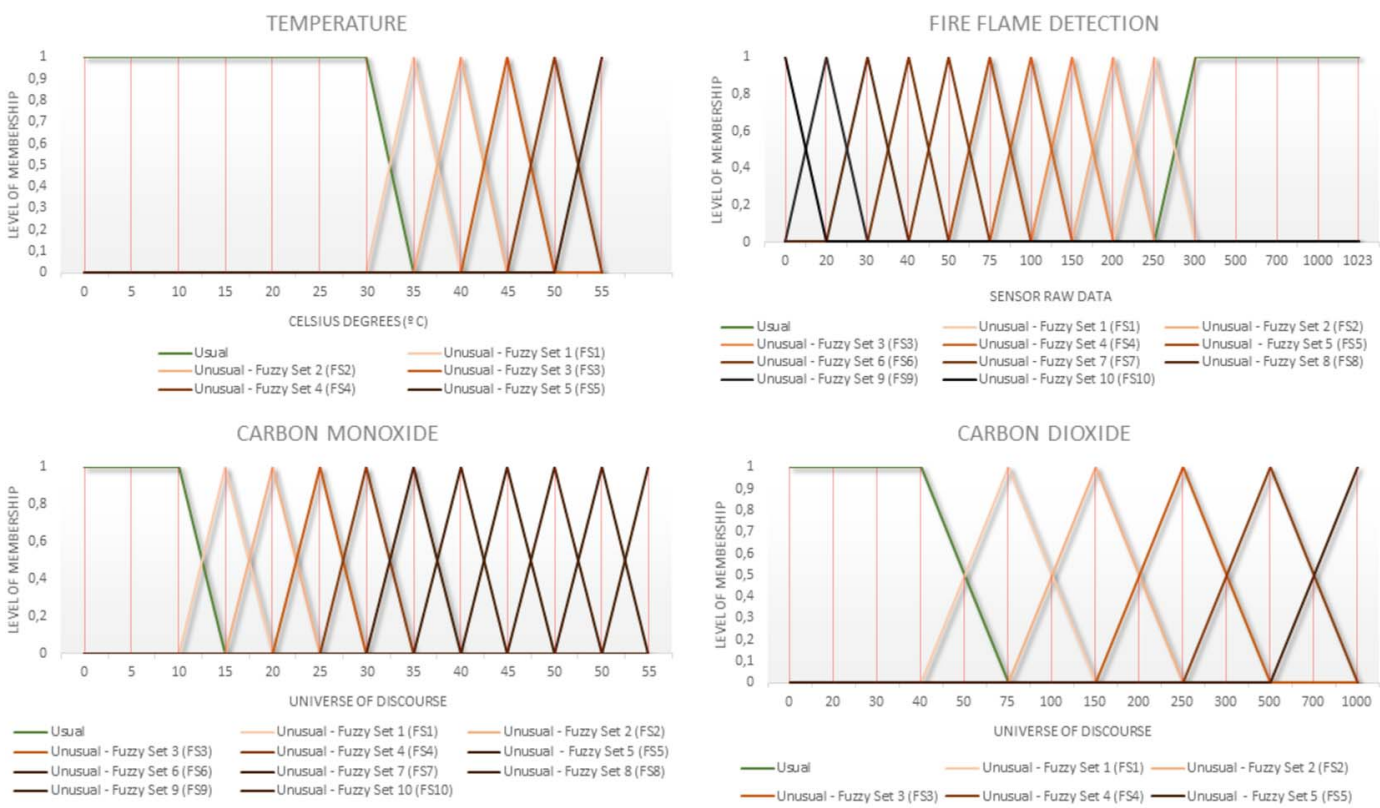

-Unusual - Fuzzy Set 3 (FS3) —Unusual - Fuzzy Set 4 (FS4) —Unusual - Fuzzy Set 5 (FS5)

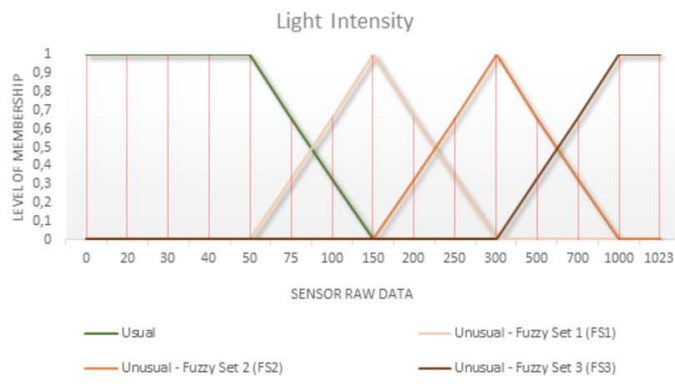

FIGURE 7. Membership functions

Thus, a dynamic membership function for every linguistic variable and every WSN is implemented and it is composed of the fuzzy set usual (usual measurements) and $n$ triangular-based unusual fuzzy sets (unusual measurements).

Figure 7 shows the membership functions defined for the environmental linguistic variables proposed for a specific WSN node. Detailed the fuzzy sets of each membership function, the calculation of the fuzzy value corresponding to a discrete input value of the proposed controller (such as a measurement of temperature) is performed according to the following equation. To this respect, the sum of the calculated levels of membership for the measurement $m_{v}$ with respect to all fuzzy sets $f_{u z z}$ set $_{v}(i)$ defined for variable $v$ is 1 .

$$
\sum \mu_{f u z z y_{-} \operatorname{set}_{v}(i)}\left(m_{v}\right)=1
$$

In addition to this, two measurement limits are defined for every input linguistic variable to manage strange environmental conditions that can result in forest fires. The aim is enhancing the classification possibilities of unusual environmental measurements and estimating probabilities of 


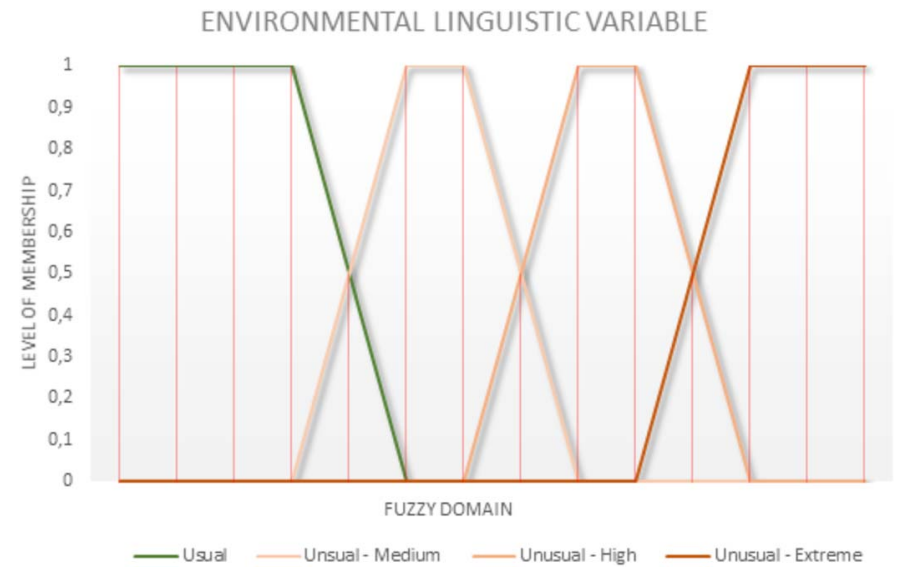

FIGURE 8. Usual, medium, high and extreme fuzzy sets

forest fire outbreaks occurrence. These limits can be defined and updated manually from the Web application. They are intended to provide a new membership function composed of 3 fuzzy sets to classify unusual measurements (medium, high and extreme measurements) or calculated by the default (see Figure 8).

Regarding the second case, the following equation can be used to calculate the amplitude of each of these trapezoidal-based fuzzy sets (trapezoid_range). Regarding a linguistic variable, the maximum value of the fuzzy domain (lim_max_fuzzy_domain), its aforementioned maxHV (maximum historical value) and its variation interval (variation_interval) are used. Inference rules also evaluate the output values depending on whether the unusual discrete measurement is fuzzified into medium. High or extreme fuzzy values (regardless of how the linguistic variable changes through triangular-based fuzzy sets).

$$
\text { trapezoid_range }=\frac{\mid \lim _{\_} \max \_ \text {fuzzy_domain }_{v}-\left(\text { maxH }_{v}+\text { variation_interval }_{v}\right) \mid}{3 \text { fuzzy sets }(\text { Medium }, \text { Hight }, \text { Extreme })}
$$

The following equation defines the trapezoidal-based fuzzy sets for medium unusual measurements. High and extreme trapezoidal fuzzy sets are defined similarly.

$$
\begin{aligned}
& t=\left(\text { maxH }+ \text { variation_interval }_{v}\right), \\
& z=((\text { maxHV}- \text { variation_interval })+\text { trapezoid_range }) \text {, } \\
& x_{\text {Medium }}\left(m_{v}\right)=\left\{\begin{array}{c}
0, m_{v}<\max H V \\
\frac{m_{v}-\max H V}{t-\max H V}, \max H V \leq m_{v} \leq t \\
1, t \leq m_{v} \leq z \\
\frac{z-m_{v}}{z-\left(z-\text { variation }_{\text {interval })}\right)},\left(z-\text { variation }_{\text {interval }}\right) \leq m_{v} \leq z
\end{array}\right.
\end{aligned}
$$

Table 3 shows a summary of the input linguistic variables. The last two columns detail the range of discrete values from which the input data of the controller can present membership levels $>0 \%$ with the usual fuzzy set and the triangular-based fuzzy sets. Thus, the maximum historical value without providing outbreak of fire detection $(\max H V)$ and the minimum historical value without providing 
Detection of Forest Fires Outbreaks

11

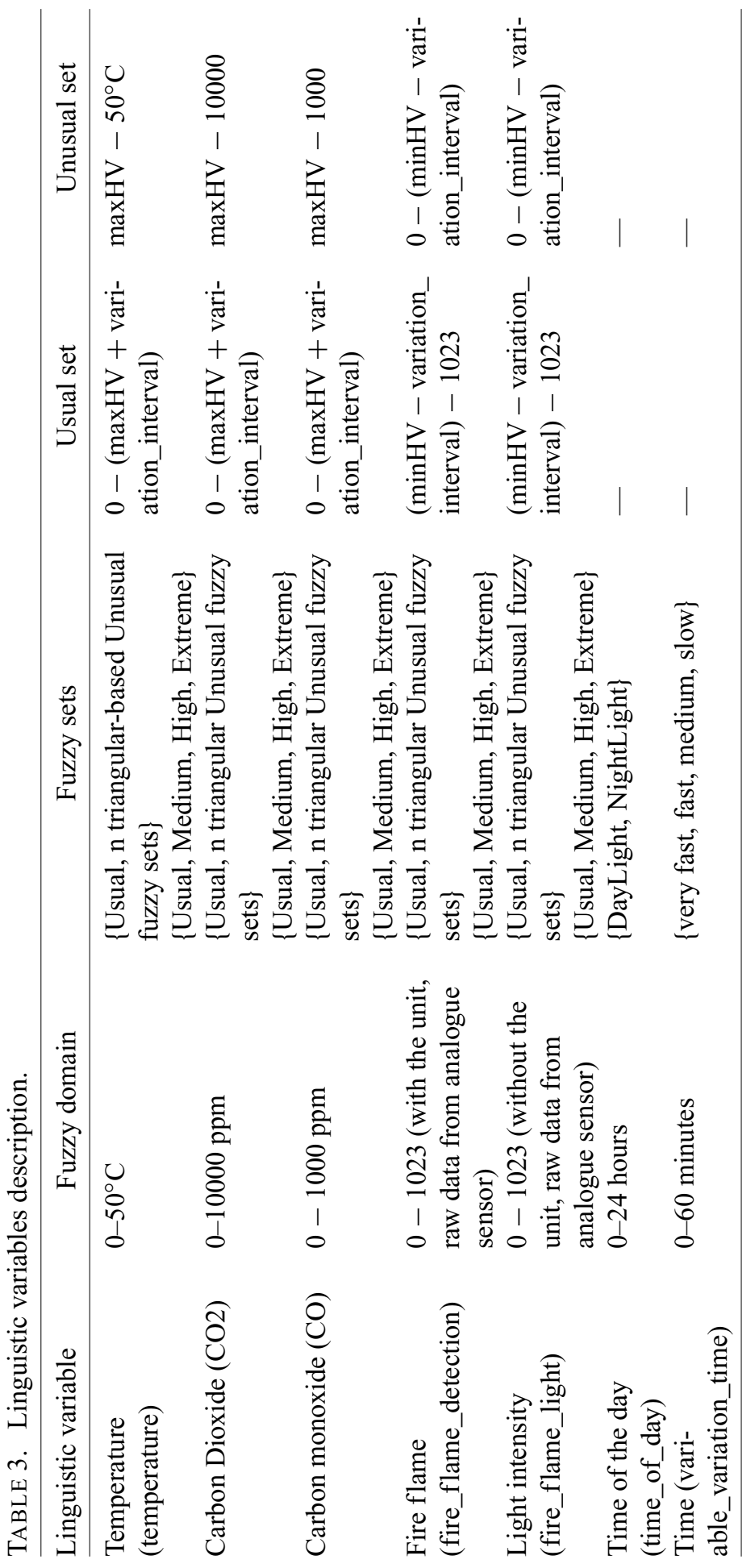




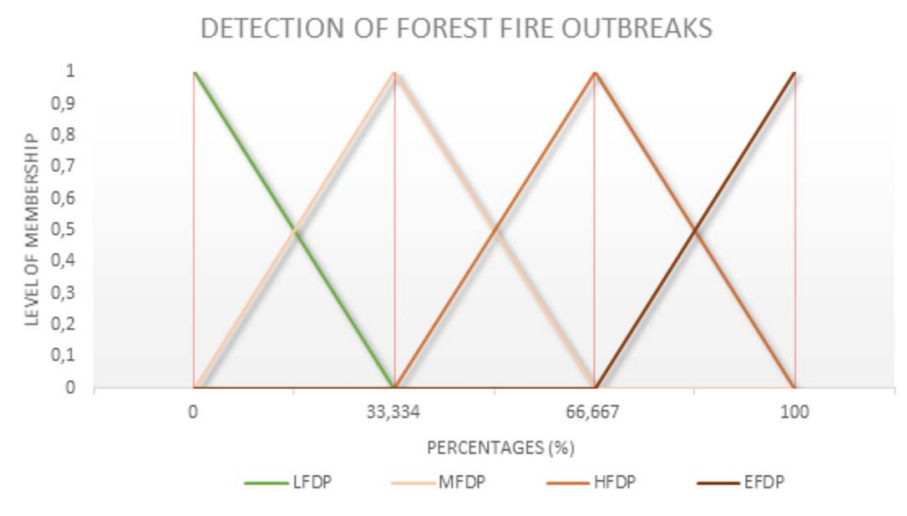

FIGURE 9. Detection of forest fire outbreaks

outbreak of fire $(\min H V)$ highlight. Likewise, the defined variation interval in case of existing an unusual environmental situation (variation_interval) stands out (detailed in Table 2).

Finally, the output linguistic variable is the probabilities of forest fire outbreaks occurrence with regard to the forest area where input environmental measurements were registered by a particular WSN node (see Figure 9). Its universe of discourse is the percentage (\%), so the outputs obtained by inference rules must range from 0 to $100 \%$ (fuzzy domain). The fuzzy sets proposed for this variable are $L F D P, M F D P, H G D P$ and EFDP.

\subsection{Knowledge base and inference rules}

The following list shows the main guidelines considered to generate the knowledge base and the inference rules of the fuzzy-based controller intended to analyse the fuzzy values obtained by fuzzification step:

1. Guideline 1. Environmental measures that present a level of membership with the fuzzy set usual involve low probability that a forest fire has been started (that means LFDP).

2. Guideline 2. When the input value of an input linguistic variable presents levels of membership $>0 \%$ with some unusual fuzzy sets (unusual environmental measurements), it is evaluated whether the fuzzy value of the last registered measurement differs from the fuzzy value of the second-last measurement, thus increasing the probability that a nearby outbreak of fire has been started. To this respect, variations between the fuzzy sets of unusual type are analysed.

3. Guideline 3. Like the previous guideline although analysing the variations between the fuzzy values according to the last environmental measurement and the average calculated (without including the last one).

4. Guideline 4. With regard to the linguistic variable of light intensity (fire_flame_light), the proposed inference rules evaluate an increase in light intensity due to a possible nearby fire flame. It is only evaluated if the fuzzy value of the linguistic variable time_of_day presents membership levels higher than $0 \%$ with the fuzzy set NightLight (so indicating night period of the current day).

5. Guideline 5. Once thresholds for classifying unusual measurements as medium, high or extreme measurements are defined (see Figure 8), the occurrence of forest fire outbreaks is estimated according to the fuzzy sets of the output linguistic variable ( $L F D P, M F D P, H F D P, E F D P$ ). 
TABLE 4 Inference rules.

\begin{tabular}{llll}
\hline $\begin{array}{l}\text { Variable__ } \\
\text { variation_time }\end{array}$ & $\begin{array}{l}\text { Fuzzy values for } \\
\text { unusual measurements }\end{array}$ & $\begin{array}{l}\text { Moves between } \\
\text { triangular-based sets }\end{array}$ & $\begin{array}{l}\text { Forest fire outbreak } \\
\text { probability }\end{array}$ \\
\hline Very low & EFDP & $>2$ moves & Extreme \\
Low & HFDP & $\leq 2$ moves & High \\
Medium & MFDP & $\geq 1$ moves & Medium \\
High & LFDP & 0 moves & Low \\
\hline
\end{tabular}

6. Guideline 6. The elapsed time (variable_variation_time) in which the fuzzy value of an input linguistic variable changes to another unusual fuzzy set that involves greater probabilities of forest fire outbreaks is evaluated.

7. Guideline 7. Moves between triangular-based unusual fuzzy sets have also been considered to evaluate the increase of forest fire risks. To this respect, quantifying and evaluating how many fuzzy sets differ from the fuzzy value of the last measurement and the corresponding fuzzy value of the second-last measurement.

Table 4 shows the inference rules defined to evaluate the elapsed time in which an environmental linguistic variable change from $i$-th triangular-based unusual fuzzy set to another increasing forest fire probabilities (Variable_variation_time and Forest fire outbreak probabilities columns, respectively). The column Fuzzy values for unusual measurements independently evaluates the existence of forest fire risks (according to Guideline 5). Finally, the column Moves between triangular-based fuzzy sets analyses forest fire outputs although independently with regard to the other columns.

The outputs obtained by the evaluation of the inference rules are aggregated in the same output set that represents all the probabilities of forest fire occurrence in the area where the environmental measurements were registered. The fuzzy values corresponding to these input environmental measurements are evaluated to trigger inference rules and obtain the final output set. Finally, centroid method is applied to convert this set into a final discrete percentage value. Thus, the probabilities of detecting a new forest fire outbreak are calculated.

\section{Conclusions and future work}

This proposal provides a forest fire detection controller intended to activate forest fire alerts by measuring environmental dynamic variables, such as polluting gases, temperature, luminosity and the presence of nearby fire flames. For this purpose, a WSN distributed throughout forest areas is proposed. This fuzzy-based controller enhances the real-time analysis of unusual environmental measurements registered by an implemented IoT prototype. A method for dynamically updating the implemented fuzzy sets is proposed to improve the interpretation of usual and unusual measurements registered in every monitored forest area. Thus, this controller is capable of specifically estimating the probabilities of forest fire outbreaks depending on every forest area. Special interest is given to ensure the integrity, confidentiality and authenticity of the transmitted measurements and the activated forest fire alerts.

Some works in progress and future lines are as follows. Regarding the fuzzy logic, the introduction of fuzzy neural networks and fuzzy decision trees on the controller is being evaluated to enhance the certainty of detecting forest fire. Other innovative IoT proposals are being studied, such as the 
integration of camera-based fire flames detection. With regard to the security scope, a survey of other low-computer encryption solutions for WSN nodes is being developed.

\section{Acknowledgements}

Research supported by the CDTI and the Ministry of Science, Innovation and Universities under Projects C2017/3-9, RTI2018-097263-B-I00 and TESIS2015010106.

\section{References}

[1] Ministerio de Agricultura, Pesca y Alimentación. Estadísticas provisionales año, 2018. https://www.mapa.gob.es/es/desarrollo-rural/estadisticas/iiff_2018_tcm30-507741.pdf. (Accessed 24 June 2019).

[2] J. I. Santos, A. E. Cesarin, C. A. Sales, M. B. Triano, P. F. R. B. Martins, A. F. Braga and N. J. Neto. Increase of atmosphere $\mathrm{CO} 2$ concentration and its effects on culture/weed interaction. International Journal of Biological, Biomolecular, Agricultural, Food and Biotechnological Engineering, 11, 419-426, 2017.

[3] I. F. Akyildiz, W. Su, Y. Sankarasubramaniam and E. Cayirci. Wireless sensor networks: a survey. Computer Networks, 38, 393-422, 2002.

[4] L. Atzori, A. Iera and G. Morabito. The internet of things: a survey. Computer Networks, 54, 2787-2805, 2010.

[5] C. C. Lee. Fuzzy logic in control systems: fuzzy logic controller. II. IEEE Transactions on Systems, Man, and Cybernetics, 20, 419-435, 1990.

[6] M. A. I. Mahmoud and H. Ren. Forest fire detection and identification using image processing and SVM. Journal of Information Processing Systems, 15, 2019.

[7] C. Yuan, Y. Zhang and Z. Liu. A survey on technologies for automatic forest fire monitoring, detection, and fighting using unmanned aerial vehicles and remote sensing techniques. Canadian Journal of Forest Research, 45, 783-792, 2015.

[8] L. Yu, N. Wang and X. Meng. Real-time forest fire detection with wireless sensor networks. In International Conference on Wireless Communications, Networking and Mobile Computing, 2005, vol. 2, pp. 1214-1217, IEEE, 2005.

[9] A. Molina-Pico, D. Cuesta-Frau, A. Araujo, J. Alejandre and A. Rozas. Forest monitoring and wildland early fire detection by a hierarchical wireless sensor network. Journal of Sensors, 2016, 2016. https://doi.org/10.1155/2016/8325845.

[10] D. Martins and H. Guyennet. Wireless sensor network attacks and security mechanisms: a short survey. In International Conference on Network-Based Information Systems, pp. 313-320, IEEE, 2010.

[11] M. Gao, Q. Wang, M. T. Arafin, Y. Lyu and G. Qu. Approximate computing for low power and security in the internet of things. Computer, 6, 27-34, 2017.

[12] K. K. Choo, S. Gritzalis and J. H. Park. Cryptographic solutions for industrial internet-ofthings: research challenges and opportunities. IEEE Transactions on Industrial Informatics, 14, 3567-3569, 2018.

[13] S. Amri, F. Khelifi, A. Bradai, A. Rachedi, M. L. Kaddachi and M. Atri. A new fuzzy logic based node localization mechanism for wireless sensor networks. Future Generation Computer Systems, 93, 799-813, 2019. 
[14] A. S. Markowski, M. S. Mannan and A. Bigoszewska. Fuzzy logic for process safety analysis. Journal of Loss Prevention in the Process Industries, 22, 695-702, 2009.

[15] P. Bolourchi and S. Uysal. Forest fire detection in wireless sensor network using fuzzy logic. In International Conference on Computational Intelligence, Communication Systems and Networks, 83-87, IEEE, 2013.

[16] P. Manjunatha, A. K. Verma and A. Srividya. Multi-sensor data fusion in cluster based wireless sensor networks using fuzzy logic method. In IEEE Region 10 and the Third International Conference on Industrial and Information Systems, 1-6, IEEE, 2008.

[17] L. S. Iliadis. Decision support system applying an integrated fuzzy model for long-term forest fire risk estimation. Environmental Modelling \& Software, 20, 613-621, 2005.

Received 1 March 2019 